\title{
Article \\ Obacunone Retards Renal Cyst Development in Autosomal Dominant Polycystic Kidney Disease by Activating NRF2
}

\author{
Zhiwei Qiu, Jinzhao He, Guangying Shao $\mathbb{D}^{-}$, Jiaqi Hu $\mathbb{D}^{-}$, Xiaowei Li, Hong Zhou, Min Li and Baoxue Yang *(i) \\ State Key Laboratory of Natural and Biomimetic Drugs, Department of Pharmacology, School of Basic Medical \\ Sciences, Peking University, Beijing 100191, China; 1410305218@pku.edu.cn (Z.Q.); 18200288030@163.com (J.H.); \\ 18210466583@163.com (G.S.); 1810301131@pku.edu.cn (J.H.); xiaowei@bjmu.edu.cn (X.L.); \\ zhouhong@bjmu.edu.cn (H.Z.); leemin@bjmu.edu.cn (M.L.) \\ * Correspondence: baoxue@bjmu.edu.cn; Tel.: +86-10-8280-5622
}

check for updates

Citation: Qiu, Z.; He, J.; Shao, G.; Hu, J.; Li, X.; Zhou, H.; Li, M.; Yang, B. Obacunone Retards Renal Cyst Development in Autosomal Dominant Polycystic Kidney Disease by Activating NRF2. Antioxidants 2022, 11, 38. https://doi.org/10.3390/ antiox11010038

Academic Editors: László Dux and Bruno Podesser

Received: 27 November 2021 Accepted: 22 December 2021 Published: 24 December 2021

Publisher's Note: MDPI stays neutral with regard to jurisdictional claims in published maps and institutional affiliations.

Copyright: (C) 2021 by the authors. Licensee MDPI, Basel, Switzerland. This article is an open access article distributed under the terms and conditions of the Creative Commons Attribution (CC BY) license (https:// creativecommons.org/licenses/by/ $4.0 /)$.

\begin{abstract}
Autosomal dominant polycystic kidney disease (ADPKD) is a common inherited disease characterized by progressive enlargement of fluid-filled cysts derived from renal tubular epithelial cells, which has become the fourth leading cause of end-stage renal diseases. Currently, treatment options for ADPKD remain limited. The purpose of this study was to discover an effective therapeutic drug for ADPKD. With virtual screening, Madin-Darby canine kidney (MDCK) cyst model, embryonic kidney cyst model and kidney-specific Pkd1 knockout mouse (PKD) model, we identified obacunone as a candidate compound for ADPKD drug discovery from a natural antioxidant compound library. In vitro experiments showed that obacunone significantly inhibited cyst formation and expansion of MDCK cysts and embryonic kidney cysts in a dose-dependent manner. In vivo, obacunone treatment significantly reduced the renal cyst development in PKD mice. Western blot and morphological analysis revealed that obacunone served as a NRF2 activator in ADPKD, which suppressed lipid peroxidation by up-regulating GPX4 and finally restrained excessive cell proliferation by downregulating mTOR and MAPK signaling pathways. Experimental data demonstrated obacunone as an effective renal cyst inhibitor for ADPKD, indicating that obacunone might be developed into a therapeutic drug for ADPKD treatment.
\end{abstract}

Keywords: obacunone; autosomal dominant polycystic kidney disease; NRF2; glutathione peroxidase; lipid peroxidation; MAPK; mTOR

\section{Introduction}

Autosomal dominant polycystic kidney disease (ADPKD) is a human inherited disease with an estimated prevalence of between one in 2500 to one in 1000 individuals [1]. It is characterized by progressive enlargement of fluid-filled cysts derived from renal tubular epithelial cells. Massive cysts gradually compress renal parenchyma, destroy the normal renal structures, and eventually cause the loss of kidney function [2]. ADPKD is mostly caused by mutations in one of two genes, $P k d 1$ or $P k d 2$, which encode the proteins polycystin-1 (PC1) or polycystin-2 (PC2), respectively [3]. The dysfunction of PC1 or PC2 leads to an abnormal decrease in intracellular $\mathrm{Ca}^{2+}$ concentration, which triggers cystogenesis by promoting excessive epithelial cell proliferation and fluid secretion [1].

With the progress of ADPKD, it eventually evolves into end-stage renal disease (ESRD), which seriously endangers the lives of patients [4,5]. Unfortunately, the therapies for ADPKD are still limited. Tolvaptan is the only drug approved by Food and Drug Administration (FDA) for ADPKD treatment at present, but the hepatotoxicity and side effects limit its application [6]. Prophylactic and symptomatic treatments remain the effective principles to retard renal cyst development. Patients with ESRD have to undergo replacement therapy [7]. These facts have brought a huge economic burden to patients and society [8]. Therefore, it is of great significance to develop drugs with a potent therapeutical effect, few side effects and a reasonable price for ADPKD treatment. 
The advantages of low toxicity and easy availability make natural compounds ideal for ADPKD therapeutic drug development. In previous studies, we had identified several natural compounds that showed effective inhibitory effects on cyst development in ADPKD experimental models, such as curcumin [9], ginkgolide B [10], Ganoderma triterpenes [11] and cardamonin [12]. The druggabilities of these compounds are still undergoing evaluation. Nevertheless, it is an effective way to screen druggable compounds based on targeting to signaling pathways that are important for the progression of ADPKD. Oxidative stress is a common pathological mechanism in the ADPKD [13]. Elevated levels of oxidative stress in renal tissues were found in both fast and slow progressing ADPKD animal models and patients [13-15]. These facts make it feasible to hunt for ADPKD therapeutic drugs from natural antioxidant compounds.

In this study, we identified obacunone from a library of natural antioxidant compounds, which showed cyst inhibitory effect in Madin-Darby canine kidney (MDCK) cyst model, embryonic kidney cyst model and kidney-specific Pkd1 knockout mouse (PKD) model. As a potent antioxidant, obacunone activated NRF2 in the early stage of ADPKD, which suppressed lipid peroxidation and finally retarded excessive cell proliferation by down-regulating mTOR and MAPK signaling pathways. Our study suggests that obacunone might be developed into a therapeutic drug for ADPKD.

\section{Materials and Methods}

\subsection{Cell Culture}

Type I Madin-Darby canine kidney (MDCK) cells (ATCC Number: CRL-2936) were cultured in a $5 \% \mathrm{CO}_{2}$ and $37^{\circ} \mathrm{C}$ humidified incubator with Dulbecco's modified Eagle's medium (DMEM) (Invitrogen, Carlsbad, CA, USA, Cat\# 12800082) containing 10\% fetal bovine serum (FBS) (Gibco, Carlsbad, CA, USA, Cat\# 10099141C), $100 \mu \mathrm{g} / \mathrm{mL}$ streptomycin and $100 \mathrm{U} / \mathrm{mL}$ penicillin (Gibco, Carlsbad, CA, USA, Cat\# 15140148). Mouse inner medullary collecting duct cells (mIMCD-3) (ATCC Number: CRL-2123) were cultured in a $5 \% \mathrm{CO}_{2}$ and $37^{\circ} \mathrm{C}$ humidified incubator with DMEM/F-12 (Invitrogen, Carlsbad, CA, USA, Cat\# 12400024) containing 10\% FBS, $100 \mu \mathrm{g} / \mathrm{mL}$ streptomycin and $100 \mathrm{U} / \mathrm{mL}$ penicillin.

\subsection{Cell Counting Kit-8 Assay}

MDCK cells were counted and seeded in quintuplicate on a 96-well plate at density of 5000 cells / well. The cells were cultured in a $5 \% \mathrm{CO}_{2}$ and $37^{\circ} \mathrm{C}$ humidified incubator with DMEM (containing 10\% FBS) for $24 \mathrm{~h}$. Then, cells in different groups were exposed to candidate compounds for $24 \mathrm{~h}$, respectively. After treatment, $100 \mu \mathrm{L} \mathrm{5 \%}$ cell counting Kit-8 (CCK-8) solution (Dojindo, Kumamoto, Japan, Cat\# CK04) was added to each well and incubated for additional $1.5 \mathrm{~h}$. Finally, the absorbance at the wavelength of $450 \mathrm{~nm}$ was measured by a microplate reader (Biotek, Santa Clara, CA, USA, MQX200). Blank wells (only containing DMEM and CCK-8) and control wells (containing untreated cells, DMEM and CCK-8) were also detected.

\subsection{MDCK Cyst Model}

MDCK cyst model was performed as previously described [11]. Briefly, MDCK cells were cultured in Type I collagen (Sigma-Aldrich, Saint Louis, MO, USA, Cat\# C2124) plated in 24-well plates at a density of 800 cells/well. DMEM/F-12 medium containing $10 \mu \mathrm{M}$ forskolin (Sigma-Aldrich, Saint Louis, MO, USA, Cat\# F6886), without or with candidate compounds, was added to each well and replaced every $12 \mathrm{~h}$.

To detect the influence of obacunone (Selleck, Houston, TX, USA, Cat\# S3784) on the formation of MDCK cysts, different concentrations of obacunone were supplied into the medium at day 0 , and the numbers of cell colonies and cysts (larger than $50 \mu \mathrm{m}$ ) in different groups were counted using a Nikon microscope (Nikon, Tokyo, Japan, ECLIPSE Ti2-U) at day 6 . The cyst formation rate was calculated by dividing the number of cysts by the number of total colonies, so as to reflect the effect of obacunone on cyst formation. Three repeating wells were set for each dose. 
To detect the influence of candidate compounds on the growth of MDCK cysts, different compounds in candidate concentration were supplied into the medium at day 4 (before micrography). And micrographs of the cysts (30/group) were taken at day 4, 6, 8, 10 and 12. Diameters of the cysts were measured using ImageJ software (National Institutes of Health, Bethesda, MD, USA).

\subsection{Embryonic Kidney Cyst Model}

Embryonic kidney cyst model was performed as described previously [11]. Briefly, kidneys from wild-type C57BL/ 6 mice at embryonic day 13.5 were isolated and placed on Transwells (Corning, Corning, NY, USA, Cat\# 3493). The embryonic kidneys were incubated with DMEM/F12 containing 2 mM L-glutamine (Aladdin, Shanghai, China, Cat\# G105425), $10 \mathrm{mM}$ HEPES, $5 \mu \mathrm{g} / \mathrm{mL}$ insulin (Sigma-Aldrich, Saint Louis, MO, USA, Cat\# I2643), $5 \mu \mathrm{g} / \mathrm{mL}$ transferrin (Sigma-Aldrich, Saint Louis, MO, USA, Cat\# T8158), $2.8 \mathrm{nM}$ sodium selenate decahydrate (Santa Cruz, Dallas, TX, USA, Cat\# sc-236924), 25 ng/mL prostaglandin E1 (Sigma-Aldrich, Saint Louis, MO, USA, Cat\# P5515), 32 pg/mL T3 (SigmaAldrich, Saint Louis, MO, USA, Cat\# T2877), $250 \mathrm{U} / \mathrm{mL}$ penicillin, $250 \mu \mathrm{g} / \mathrm{mL}$ streptomycin and $100 \mu \mathrm{M}$ 8-Br-cAMP (Sigma-Aldrich, Saint Louis, MO, USA, Cat\# B5386) (the blank group did not contain 8-Br-cAMP). Different compounds in candidate concentration were supplied to the medium at day 0 , and medium was replaced every $12 \mathrm{~h}$. Kidneys were photographed using a Nikon microscope at day 0, 2, 4 and 6. Cyst index was calculated by comparing the total cyst area to total kidney area using the ImageJ software (National Institutes of Health, Bethesda, MD, USA).

\subsection{Animals}

$P k d 1^{f l o x / f l o x}$ mice (from the Yale PKD Center) and Ksp-Cre transgenic mice (from the University of Texas Southwestern O'Brien Center) on C57BL/ 6 background were generated as described previously [16]. Kidney-specific $P k d 1$ knockout mice (with $P k d 1^{\text {flox/flox }}$; Ksp-Cre genotype) were generated by cross-breeding Pkd1 flox/flox mice with Ksp-Cre mice. Neonatal mice (1 day old) were genotyped by genomic PCR. In order to explore the therapeutical effect of obacunone on ADPKD, $100 \mathrm{mg} / \mathrm{kg}$ obacunone (dissolved in saline with 1/1000 DMSO) was injected intraperitoneally every day from postnatal day 1 to day 4 . At postnatal day 5, kidneys were removed, weighed and fixed for histologic examination. In order to explore the activation effect of obacunone on NRF2 in the early stage of ADPKD, $100 \mathrm{mg} / \mathrm{kg}$ obacunone was injected intraperitoneally every day from postnatal day 1 to day 2 . At postnatal day 3 , kidneys were removed, weighed and fixed for histologic examination.

\subsection{Histology}

Kidneys were collected and processed as described previously [12]. Briefly, kidneys were fixed by $4 \%$ paraformaldehyde (Sigma-Aldrich, Saint Louis, MO, USA, Cat\# 158127), gradiently dehydrated by alcohol (Tong Guang, Beijing, China, Cat\# 104022) and embedded in paraffin (Leica, Wetzlar, Germany, Cat\# 39601095). $5 \mu \mathrm{m}$ sections were cut and stained with hematoxylin (Amresco, Radnor, PA, USA, Cat\# 0701) and eosin (Amresco, Radnor, PA, USA, Cat\# 0109). Kidney sections were photographed using a Nikon microscope. Cyst index was calculated by comparing the total cyst area to total kidney area using the ImageJ software (National Institutes of Health, Bethesda, MD, USA).

\subsection{Immunofluorescence Staining}

For kidney tissue, kidneys were fixed by $4 \%$ paraformaldehyde, gradiently dehydrated by sucrose (Aladdin, Shanghai, China, Cat\# S112228) and embedded in optimal cutting temperature compound (SAKURA, Tokyo, Japan, Cat\# 4583). $5 \mu \mathrm{m}$ sections were cut. $5 \%(w / v)$ bovine serum albumin was used to block the sections at room temperature for $1 \mathrm{~h}$. Then, the sections were incubated with primary antibodies against Ki-67 (Abcam, Cambridge, UK, Cat\# ab15580, 1:500 dilution) at $4{ }^{\circ} \mathrm{C}$ overnight. The next day, sections were 
washed 3 times and incubated with Cy3-conjugated Affinipure goat anti-rabbit $\operatorname{IgG}(\mathrm{H}+\mathrm{L})$ (Proteintech, Wuhan, China, Cat\# SA00009-2, 1:100 dilution) secondary antibodies or Lotus tetragonolobus lectin (LTL, Vector Laboratories, Burlingame, CA, USA, Cat\# FL-1321, 1:400 dilution) and Dolichos biflorus agglutinin (DBA) (Vector Laboratories, Burlingame, CA, USA, Cat\#, RL-1032, 1:400 dilution) for 1 h. DAPI (Sigma, Saint Louis, MO, USA, Cat\# D9542) was used to stain nuclei. Images were captured with a Nikon fluorescence microscope (Nikon, Tokyo, Japan, ECLIPSE Ti2-U). The unit areas of Ki-67 positive cells were calculated using ImageJ software (National Institutes of Health, Bethesda, MD, USA).

For mIMCD-3 cells, cells were seeded into glass bottom dish (Cellvis, Mountain View, CA, USA, Cat\# D35-10-1-N). After stimulation without or with forskolin or/and obacunone, $4 \%$ paraformaldehyde was added to fix the cells for $15 \mathrm{~min} .5 \%(\mathrm{w} / \mathrm{v})$ bovine serum albumin was used to block the sections at room temperature for $1 \mathrm{~h}$. Then, the cells were incubated with primary antibodies against NRF2 (ABclonal, Wuhan, China, Cat\# A0674, 1:200 dilution) and $\beta$-actin (ABclonal, Wuhan, China, Cat\# AC004, 1:200 dilution) at $4{ }^{\circ} \mathrm{C}$ overnight. The next day, cells were washed 3 times and incubated with fluorescein (FITC)-conjugated affinipure goat anti-mouse IgG(H+L) (Proteintech, Wuhan, China, Cat\# SA00003-1, 1:100 dilution) and Cy3-conjugated affinipure goat anti-rabbit IgG(H+L) (Proteintech, Wuhan, China, Cat\# SA00009-2, 1:100 dilution) secondary antibodies. DAPI (Sigma, Saint Louis, MO, USA, Cat\# D9542) was used to label nuclei. Images were captured by a Nikon confocal microscope (Nikon, Tokyo, Japan, TiE-A1 plus).

\subsection{Western Blot Analysis}

Total kidney protein was extracted using RIPA lysis buffer (Applygen, Beijing, China, Cat\# C1053) containing 4\% protease inhibitor cocktail (Roche, South San Francisco, CA, USA, Cat\# 11873580001) and 1\% protein phosphatase inhibitor (Applygen, Beijing, China, Cat\# P1260). Nucleoprotein was extracted using a nucleoprotein extraction kit (Bestbio, Shanghai, China, Cat\# BB-3102).

Protein samples were separated based on molecular weight through SDS-polyacrylamide gel electrophoresis and transferred to polyvinylidene difluoride membranes (PALL, Beijing, China, Cat\# BSP0161). The membranes were blocked with blocking buffer (TBS, 0.1\% Tween$20,5 \%$ non-fat milk or $2 \%$ BSA) for $2 \mathrm{~h}$ at room temperature and incubated with primary antibodies against NRF2 (ABclonal, Wuhan, China, Cat\# A0674, 1:1000 dilution), Lamin B1 (ABclonal, Wuhan, China, Cat\# A1910, 1:1000 dilution), 4-HNE (Bioss, Beijing, China, Cat\# bs-6313R, 1:1000 dilution), GPX4 (ABclonal, Wuhan, China, Cat\# A13309, 1:1000 dilution), H-Ras (ABclonal, Wuhan, China, Cat\# A19619, 1:1000 dilution), B-Raf (ABclonal, Wuhan, China, Cat\# A15033, 1:1000 dilution), p-ERK (ABclonal, Wuhan, China, Cat\# AP0974, 1:1000 dilution), ERK (ABclonal, Wuhan, China, Cat\# A16686, 1:1000 dilution), p-mTOR (Cell Signaling Technology, Danvers, MA, USA, Cat\# 5536S, 1:1000 dilution), mTOR (Cell Signaling Technology, Danvers, MA, USA, Cat\# 2983, 1:1000 dilution), p-S6 (ABclonal, Wuhan, China, Cat\# AP0537, 1:1000 dilution), S6 (ABclonal, Wuhan, China, Cat\# A6058, 1:1000 dilution), PCNA (Cell Signaling Technology, Danvers, MA, Cat\# 2586, 1:1000 dilution), $\beta$-actin (ABclonal, Wuhan, China, Cat\# AC004, 1:10,000 dilution) at $4{ }^{\circ} \mathrm{C}$, respectively. Afterwards, HRP-conjugated goat anti-rabbit IgG (H+L) (Immunoway, Suzhou, China, Cat\# RS0002) or HRP-conjugated goat anti-mouse IgG (Easybio, Beijing, China, Cat\# BE0102) were incubated for $60 \mathrm{~min}$ at room temperature. The ECL kit (Meilunbio, Dalian, China, Cat\# MA0186) was used to detect protein expression signal intensity through a chemiluminescence detection system (Syngene, Frederick, MD, USA, GeneGnome XRQ). Relative protein expression levels were quantified by the ImageJ software (National Institutes of Health, Bethesda, MD, USA).

\subsection{GSH-Px, GSH, and MDA Assay}

The activity of GSH-Px and content of GSH and MDA in the kidney were detected using commercial kits as follows: glutathione peroxidase (GSH-PX) assay kit (Nanjing Jiancheng, Nanjing, China, Cat\# A005-1-2), reduced glutathione (GSH) assay kit (Nanjing 
Jiancheng, Nanjing, China, Cat\# A006-2-1), malondialdehyde (MDA) assay kit (Nanjing Jiancheng, Nanjing, China, Cat\# A003-1-2). Detection procedure is carried out in strict accordance with the protocols provided by the kits.

\subsection{Statistical Analyses}

All results were represented as Mean \pm SEM. Statistical analyses were performed with GraphPad Prism 8.0 software. Data were first analyzed with the D'Agostino-Pearson omnibus normality test to verify their distribution. Parametric tests were performed where data obeyed gaussian distribution. Data involving in two groups were analyzed using two-tailed student's $t$ test. When more than two experimental groups were compared, the data were analyzed using one-way ANOVA with Tukey multiple comparison tests. Data that did not obey gaussian distribution were analyzed using the Kruskal-Wallis test. $p$-value $<0.05$ was considered to be statistically significant.

\section{Results}

\subsection{Obacunone Is Identified as an Inhibitor of Renal Cysts}

By a virtual screening based on the effect on oxidative stress response-related signaling pathways, such as KEAP1/NRF2, NOX4 and PERK, 10 candidate compounds (Y1-Y10) were selected from a natural compound library at Selleck. CCK-8 assay showed that $12.5 \mu \mathrm{M}$ Y 8 and $\mathrm{Y} 9$ significantly reduced MDCK cell viability (Figure 1A), which were excluded in the further studies due to their cytotoxicity.

The remaining eight compounds $(12.5 \mu \mathrm{M})$ were investigated in a MDCK cyst model to detect their cyst inhibitory effect. MDCK cells form cysts in type I collagen with $10 \mu \mathrm{M}$ forskolin stimulation, which is widely used as an in vitro model of ADPKD. As shown in Figure 1B,C, $12.5 \mu \mathrm{M}$ Y1, Y4, Y5, Y6 or Y10 significantly inhibited the expansion of MDCK cysts. These five compounds $(12.5 \mu \mathrm{M})$ were further detected with an embryonic kidney cyst model to evaluate the cyst inhibitory activity at the organ level. E13.5 embryonic kidneys cultured in transwell form cysts with $100 \mu \mathrm{M}$ 8-Br-cAMP stimulation, which is used as an ex vivo model of ADPKD. As shown in Figure 1D,E, Y4, Y6 or Y10 stimulation significantly retarded cyst development in embryonic kidney. However, Y4 inhibited the growth of embryonic kidneys (Figure 1D,F), indicating its toxic effect on kidney development.

In vivo inhibiting effects of $\mathrm{Y} 6$ and $\mathrm{Y} 10$ on renal cyst development were determined in a kidney-specific $P k d 1$ knockout mouse (Pkd1 $\left.{ }^{f l o x} f f l o x ; K s p-C r e, P K D\right)$ model. PKD mice develop acutely progressive ADPKD, in which renal cysts appear at postnatal day 1 and progressively enlarge, which causes death of the animal around postnatal day 14 [12]. As shown in Figure 1G, PKD kidneys were much larger than wild-type ones. Y10 $(100 \mathrm{mg} /(\mathrm{kg} \cdot \mathrm{d})) \mathrm{re}-$ duced the kidney volume of PKD mice without affecting kidney development in wild-type mice. These results indicate that $\mathrm{Y} 10$ has potent cyst inhibition activity and little cytotoxicity. The chemical structure of Y10, which is called obacunone, is shown in Figure 1H. 

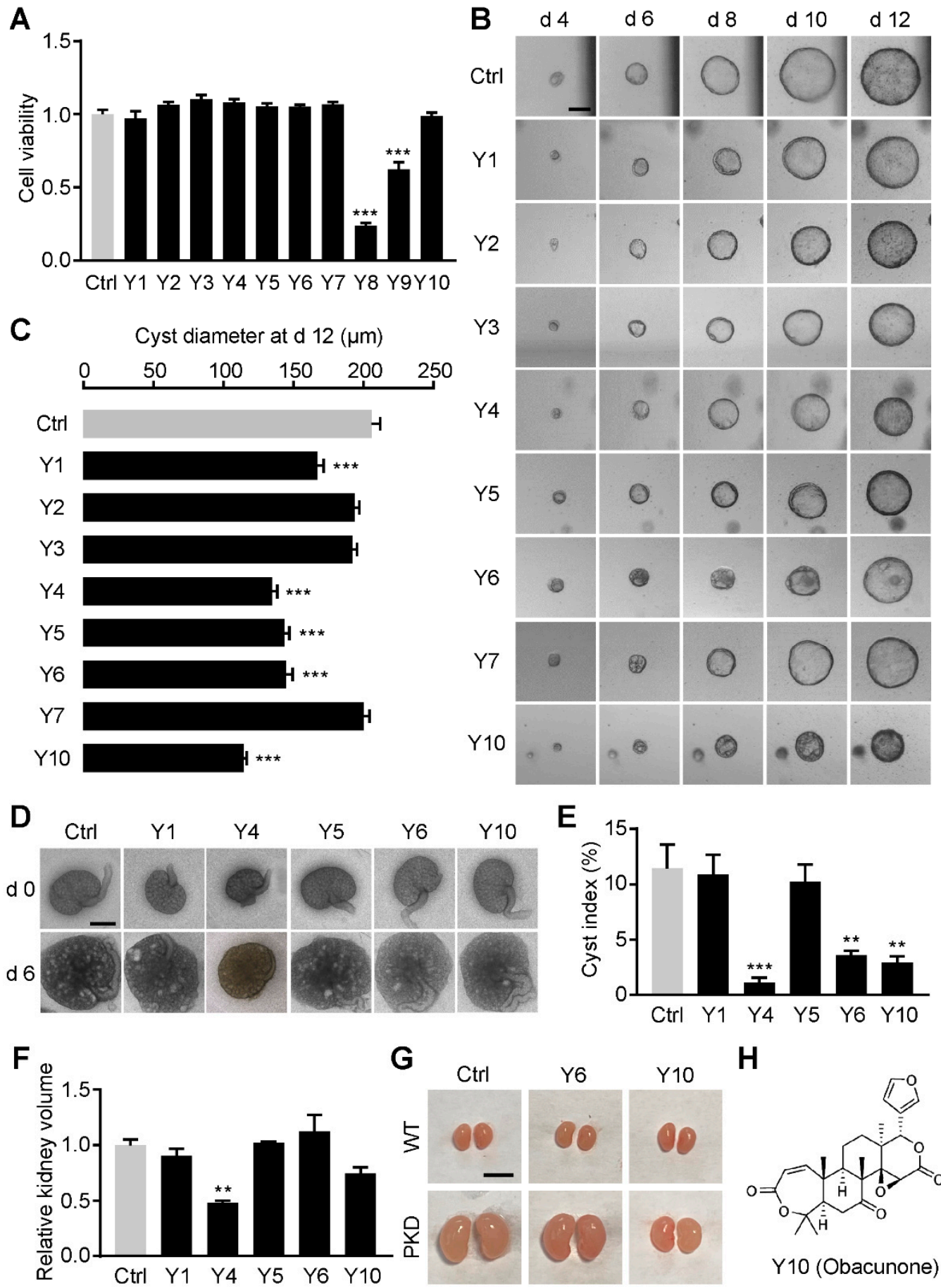

H

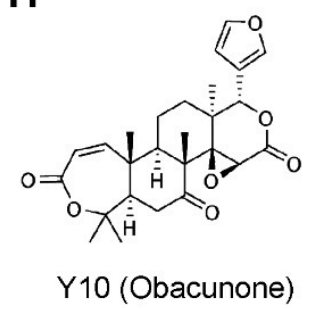

Figure 1. Identification of obacunone as a potent renal cyst inhibitor. (A) Cytotoxicity of $12.5 \mu \mathrm{M}$ candidate compounds on MDCK cells. $n=5$. (B) Representative images of MDCK cysts treated without (Ctrl) or with $12.5 \mu \mathrm{M}$ candidate compounds. Bar $=100 \mu \mathrm{m}$. (C) Diameters of MDCK cysts at day 12. $n=30$. (D) Representative images of embryonic kidneys. Bar $=500 \mu \mathrm{m}$. (E) Cyst indices of embryonic kidneys at day 6. $n=6$. (F) Volumes of embryonic kidneys at day 6. $n=6$. (G) Representative images of kidneys from wild-type (WT) or kidney specific Pkd1 knockout mice (PKD) treated without or with $100 \mathrm{mg} /(\mathrm{kg} \cdot \mathrm{d})$ candidate compounds. Bar $=0.5 \mathrm{~cm}$. $(\mathbf{H})$ Chemical structure of Y10. Data are presented as means \pm SEM. ${ }^{* *} p<0.01,{ }^{* * *} p<0.001$, versus Ctrl. 


\subsection{Obacunone Dose-Dependently Inhibits MDCK Cyst Formation and Enlargement}

CCK-8 assay showed that obacunone had no significant cytotoxicity to MDCK cells at concentration of $50 \mu \mathrm{M}$ and below (Figure 2A). We first evaluated the effect of obacunone on MDCK cyst formation. MDCK cells were incubated in type I collagen without or with obacunone at $3.12,12.5$ or $50 \mu \mathrm{M}$ from day 0 to day 6 . At day 6 , the numbers of total colonies (including both cell colonies and cysts) were similar among all groups (Figure 2B). However, numbers of cysts (diameter larger than $50 \mu \mathrm{m}$ ) were reduced by obacunone treatment in a dose-dependent manner with inhibitory rates of $\sim 7.3 \%, \sim 12.2 \%$ and $\sim 46.9 \%$, respectively (Figure $2 \mathrm{C}$ ), indicating that obacunone significantly inhibited the formation of MDCK cysts.

To evaluate the effect of obacunone on the cyst enlargement, MDCK cysts were treated without or with obacunone from day 5 to day 12. It was found that cyst growth was inhibited by obacunone in a dose-dependent manner (Figure 2D,E). Interestingly, when obacunone was washed out at day 8, inhibited MDCK cysts regrew again (Figure 2F), suggesting that the inhibitory effect of obacunone on MDCK cyst growth was reversible.

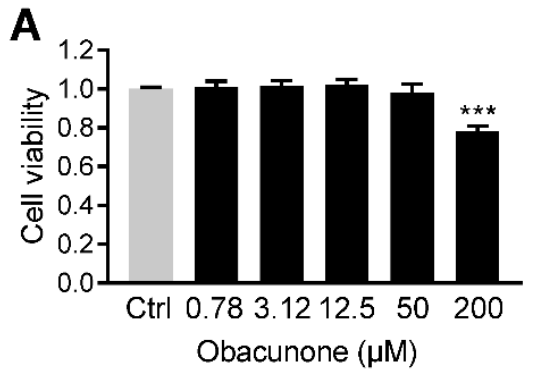

D

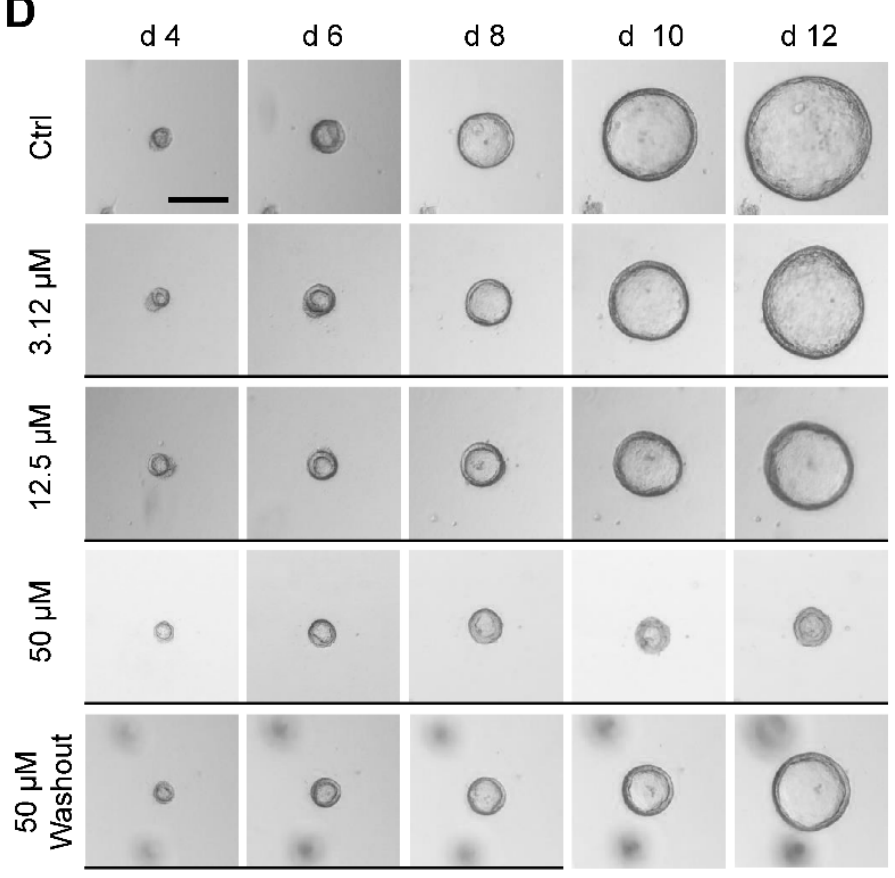

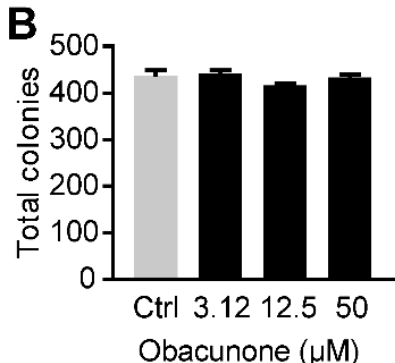

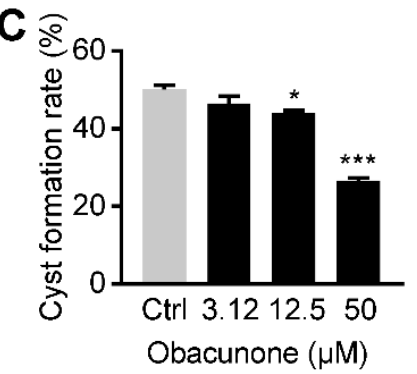

E

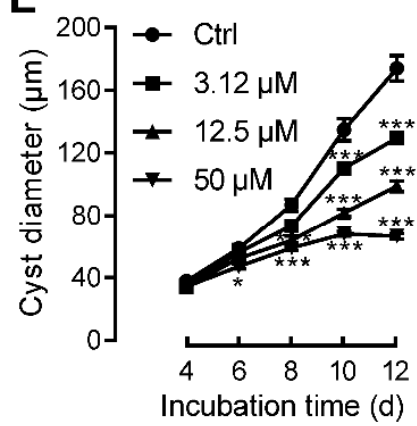

$\mathbf{F}$

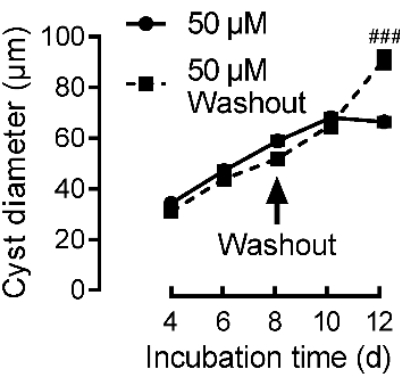

Figure 2. Inhibitory effect of obacunone on MDCK cyst formation and expansion. (A) Viability of MDCK cells treated without (Ctrl) or with obacunone. $n=5$. (B) Number of total colonies in MDCK cyst model. $n=3$. (C) Cyst formation rate. $n=3$. (D) Representative images of MDCK cysts. Narrow black lines indicate obacunone treatment. Bar $=100 \mu \mathrm{m}$. (E) Diameters of MDCK cysts showing dose response of obacunone. $n=30$. (F) Diameter of MDCK cysts showing reversible effect of obacunone. Arrow indicates the time of washout obacunone. $\mathrm{n}=30$. Data are presented as means \pm SEM. ${ }^{*} p<0.05,{ }^{* * *} p<0.001$ versus Ctrl. ${ }^{\# \#} p<0.001$ versus $50 \mu \mathrm{M}$ obacunone treatment without washout. 


\subsection{Obacunone Inhibits Cyst Development in Embryonic Kidney}

To confirm the inhibitory effect of obacunone at the organ level, we detected cyst development in the embryonic kidney cyst model. Experimental results showed that obacunone dose-dependently inhibited the renal cysts enlargement, which is consistent with the observation in the MDCK cyst model. Similarly, the inhibitory effect of $50 \mu \mathrm{M}$ obacunone on the cyst growth was abolished when obacunone was washed out at day 4 (Figure 3A,B), confirming the reversible therapeutical effect of obacunone on ADPKD. However, obacunone had no adverse effect on the development of embryonic kidneys, as the obacunone-treated embryonic kidneys had similar volumes as the 'blank' (i.e., no 8-Br-cAMP and no obacunone) and 'control' (i.e., no obacunone) groups (Figure 3A,C).

A

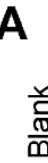

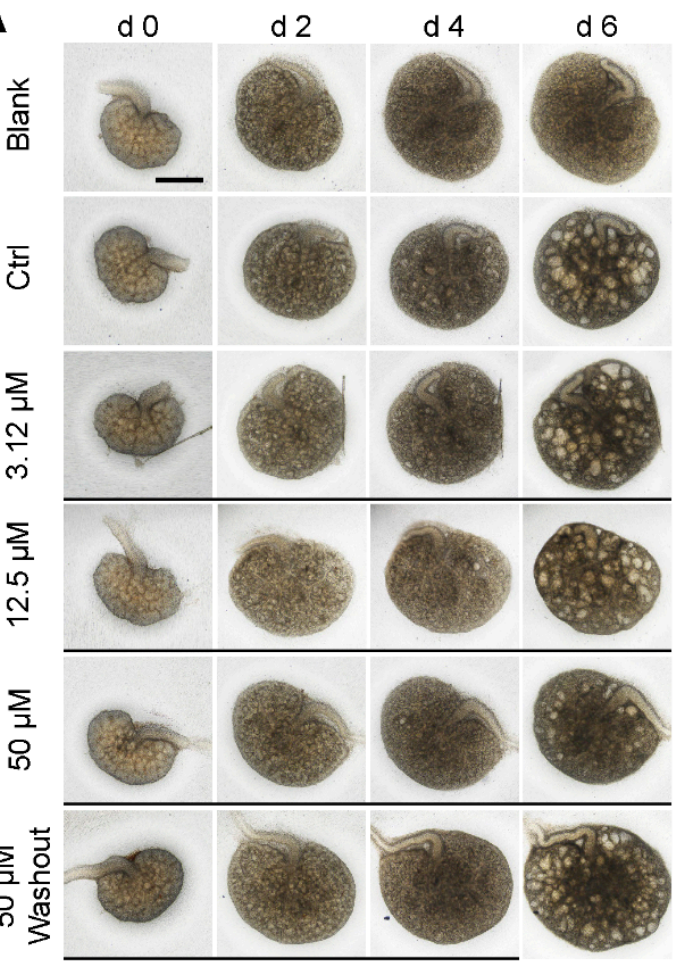

B
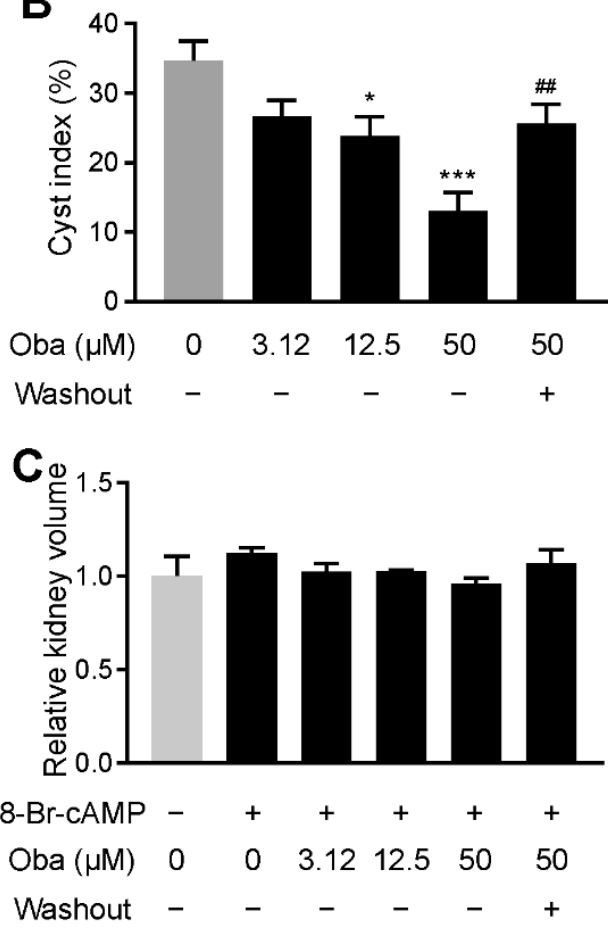

Figure 3. Inhibitory effect of obacunone on the enlargement of embryonic kidney cyst. (A) Representative images of embryonic kidneys treated without (Ctrl) or with obacunone. Narrow black lines indicate obacunone treatment. Bar $=500 \mu \mathrm{m}$. (B) Cyst indices (cyst area to embryonic kidney area). (C) Relative kidney volumes of embryonic kidneys on day 6. Data are presented as means \pm SEM. $\mathrm{n}=6{ }^{*} p<0.05,{ }^{* * *} p<0.001$ versus Ctrl. ${ }^{\# \#} p<0.01$ versus $50 \mu \mathrm{M}$ obacunone treatment.

\subsection{Obacunone Retards Renal Cyst Development in PKD Mice}

We further investigated the cyst inhibitory effect of obacunone in vivo. Treatment with obacunone $(100 \mathrm{mg} / \mathrm{kg}$, injected intraperitoneally every day from postnatal day 1 to day 4$)$ did not affect the size, growth and behavior of mice (Figure 4A), but significantly retarded the enlargement of bilateral kidneys of PKD mice (Figure 4B,C). Kidney sections showed that obacunone significantly reduced the kidney cyst indices of PKD mice (Figure 4D,E). Moreover, labeling collecting ducts by DBA and proximal tubules by LTL with immunostaining, we found that the renal cysts were mainly formed in collecting ducts rather than proximal tubules (Figure $4 \mathrm{~F}$ ). 


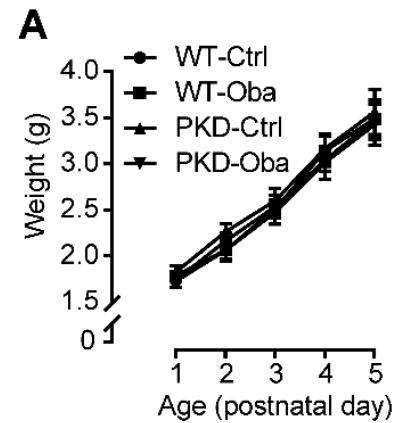

D

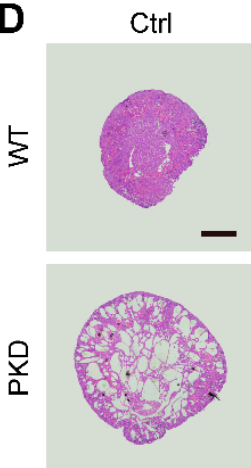

$\mathbf{F}$
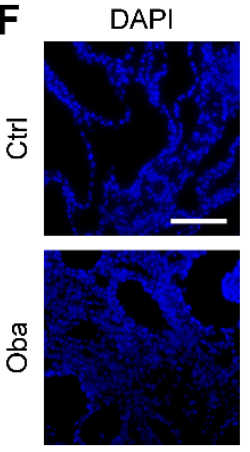

B

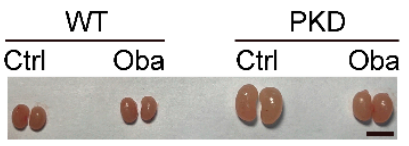

C

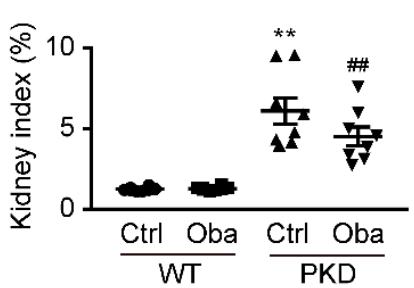

E

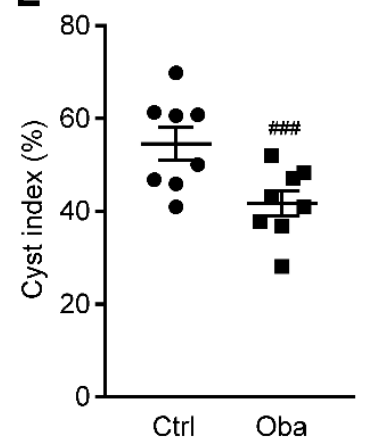

DBA

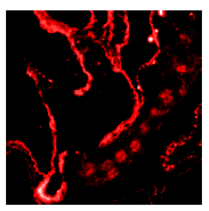

Merged
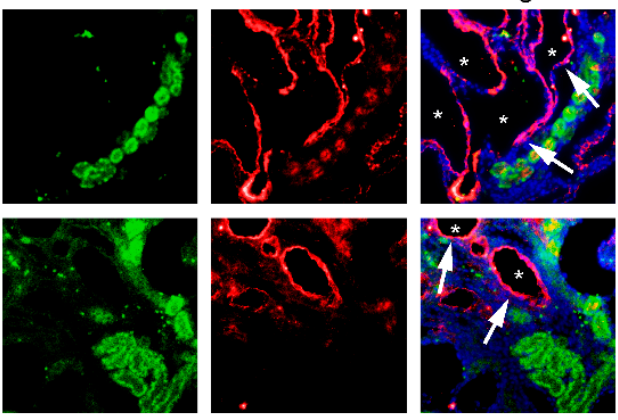

Figure 4. Inhibitory effect of obacunone on cyst development in PKD mice. (A) Weight curves of mice. (B) Representative images of the kidneys. Bar $=1 \mathrm{~cm}$. (C) Kidney indices. (D) Hematoxylinand eosin-stained pictures of kidneys. Bar $=400 \mu \mathrm{m}$. (E) Cyst indices. (F) LTL (green), DBA (red) and DAPI (blue) fluorescence staining of PKD kidneys. Arrows indicate the collecting ducts, asterisks represent the cyst area. Bar $=50 \mu \mathrm{m}$. Data are presented as means \pm SEM. $\mathrm{n}=8$. ${ }^{* *} p<0.01$ versus WT-Ctrl. ${ }^{\#} p<0.01,{ }^{\# \#} p<0.001$ versus PKD-Ctrl.

\subsection{Obacunone Activates NRF2 in the Early Stage of ADPKD}

Previous studies have reported that obacunone is an effective activator of NRF2 [17-20] that plays an important role in ROS scavenging [21-23]. NRF2 knockout promotes the development of renal cysts in ADPKD mouse models [24]. Therefore, we investigated the effect of obacunone on NRF2 activation. Out of our expectation, although obacunone promoted nuclear translocation of NRF2 in FSK-stimulated mIMCD-3 cells (Figure 5A,B), obacunone treatment for four days did not up-regulate intranuclear NRF2 in PKD kidneys (data not shown). Considering that oxidative stress response usually occurs before cyst formation in ADPKD [15], and the activity of NRF2 often changes dynamically during the disease progression [25], we speculated that obacunone might activate NRF2 in the early stage of ADPKD.

To verify our hypothesis, we explored the activation effect of obacunone on NRF2 in the early stage of ADPKD in vivo. PKD mice were treated with obacunone $(100 \mathrm{mg} / \mathrm{kg}$, injected intraperitoneally every day) from postnatal day 1 to day 2 . The kidneys were sampled at postnatal day 3 and the expression level of intranuclear NRF2 was detected immediately. 
The experimental results showed that short-term obacunone treatment significantly upregulated the expression of NRF2 in the renal cell nucleus of PKD mice (Figure 5C,D), which indicates that obacunone activates NRF2 in the early stage of ADPKD.
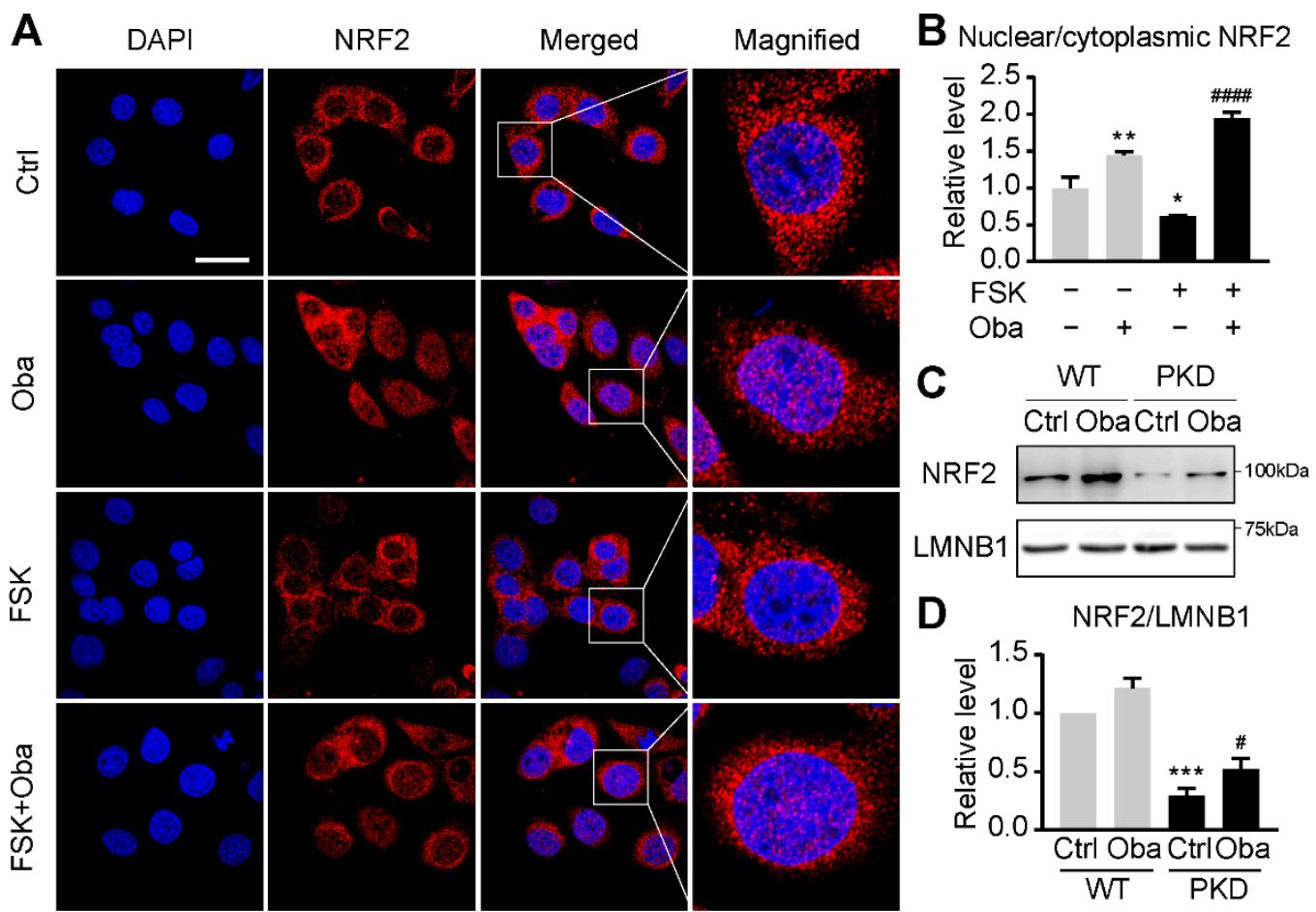

Figure 5. Effect of obacunone on NRF2 activity. (A) Immunofluorescence of NRF2 (red) and DAPI (blue) staining in mIMCD-3 cells treated without (Ctrl) or with forskolin (FSK) or obacunone (Oba). Bar $=10 \mu \mathrm{m}$. (B) Quantification of fluorescence intensities of NRF2 as in (A). $\mathrm{n}=12$ cells. (C) Representative Western blots of nuclear NRF2 in the kidneys of wild-type (WT) and PKD mice (PKD) treated without or with obacunone. (D) Quantification of nuclear NRF2 expression levels as in (C). Data are presented as means \pm SEM. $\mathrm{n}=6 .{ }^{*} p<0.05,{ }^{* *} p<0.01$ versus Ctrl. ${ }^{\# \# \#} p<0.0001$ versus FSK. ${ }^{* * *} p<0.001$ versus WT-Ctrl. ${ }^{\#} p<0.05$ versus PKD-Ctrl.

\subsection{Obacunone Suppresses Lipid Peroxidation in ADPKD}

To further explore the effect of obacunone-activated NRF2 on the development of ADPKD, we detected the expression of the NRF2 target gene glutathione peroxidase 4 (GPX4) in PKD mice at postnatal day 5. Western blot results showed that obacunone significantly up-regulated the GPX4 expression in PKD kidneys (Figure 6A,B), which was accompanied by an enhancement of glutathione peroxidase (GSH-Px) activity (Figure 6C). The decrease of glutathione (the substrate of GPX4) in ADPKD was also reversed by obacunone treatment (Figure 6D).

Previous studies have confirmed that GPX4 is a key factor in regulating lipid peroxidation [26], which plays a catalytic role in promoting the ADPKD progression [14]. Hence, we investigated the effect of obacunone on lipid peroxidation in PKD mice. As expected, the levels of 4-hydroxy-2-nonenal (4-HNE)-modified protein (reflecting the content of 4$\mathrm{HNE}$ ) (Figure 6A,E) and content of malondialdehyde (MDA) (Figure 6F) were increased in kidneys from control PKD mice and reduced with obacunone treatment. 
A

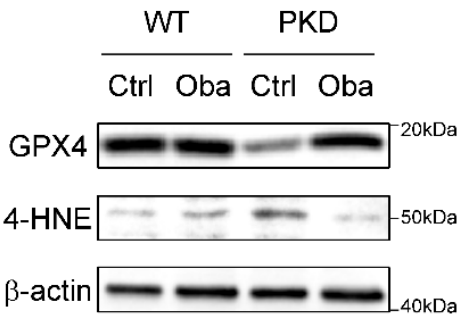

D

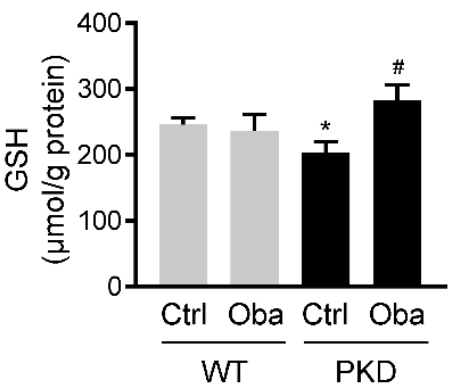

B

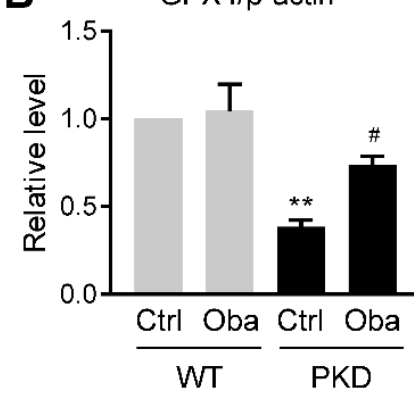

E

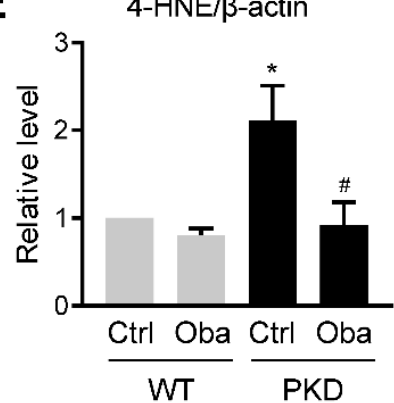

C

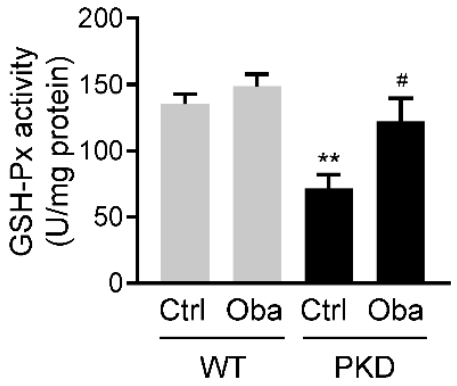

F

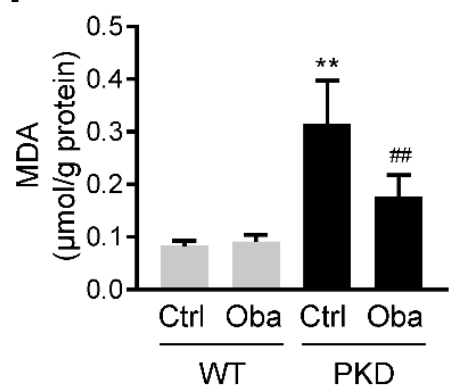

Figure 6. Inhibition effect of obacunone on lipid peroxidation. (A) Representative Western blots of GPX4 and 4-HNE in the kidneys of wild-type (WT) and PKD (PKD) mice treated without (Ctrl) or with obacunone (Oba). (B) Quantification of GPX4 expression levels. (C) GSH-Px activity. (D) GSH content. (E) Quantification of 4-HNE expression levels. (F) MDA content. Data are presented as means \pm SEM. $\mathrm{n}=6$. ${ }^{*} p<0.05,{ }^{* *} p<0.01$ versus WT-Ctrl. ${ }^{\#} p<0.05,{ }^{\# \#} p<0.01$ versus PKD-Ctrl.

3.7. Obacunone Inhibits Abnormal Cell Proliferation by Down-Regulating mTOR and MAPK Signaling Pathways

Large amounts of lipid peroxides (LPOs), such as MDA and 4-HNE [27], have been reported as the activators of mTOR [28,29] and MAPK [30-32] signaling pathways, which are involved in the ADPKD progression. Therefore, we investigated the effect of obacunone on these two signaling pathways. It was found that obacunone significantly inhibited the expression of H-Ras, B-Raf and phosphorylation of ERK, mTOR and S6 in the PKD kidneys (Figure 7A-G), indicating the down-regulation of mTOR and MAPK signaling pathways. Moreover, the expression of PCNA (Figure 7H,I) and Ki-67 (Figure 7J,K) was also down-regulated by obacunone, suggesting the retardation of abnormal cell proliferation in the PKD kidneys. 
A

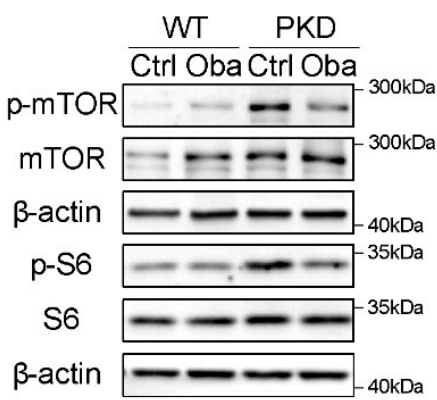

D

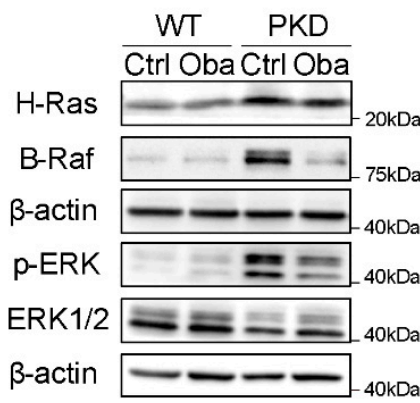

G

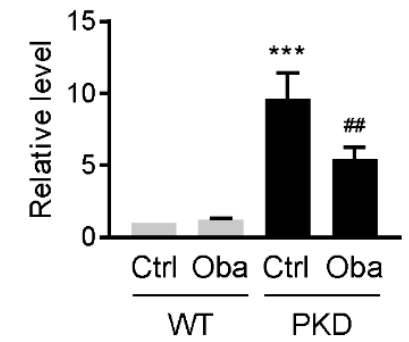

J

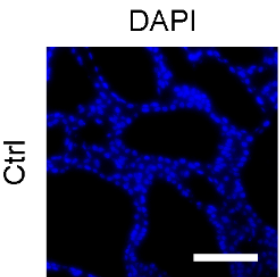

Ki-67
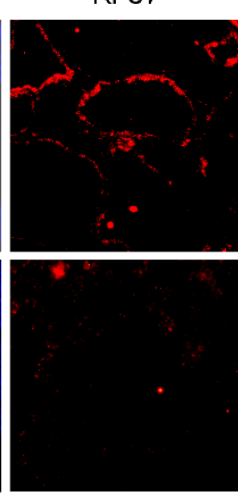

B

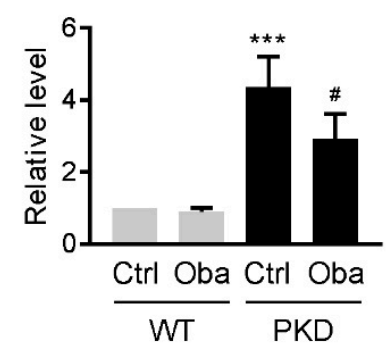

E

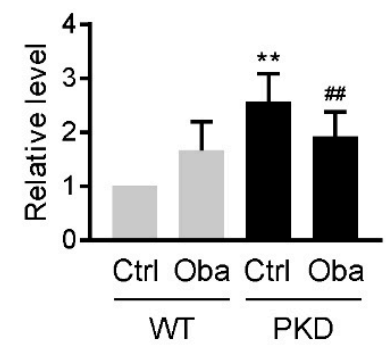

C $\mathrm{p}-\mathrm{S} 6 / \mathrm{s} 6$

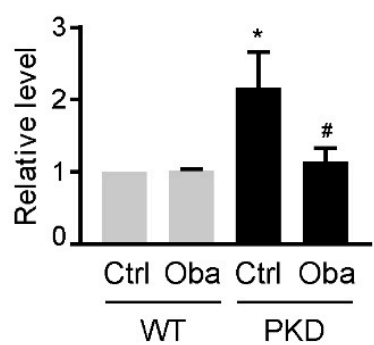

F

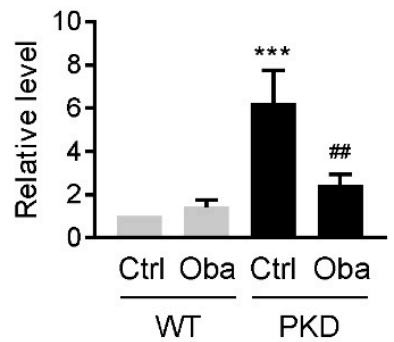

I

PCNA/B-actin
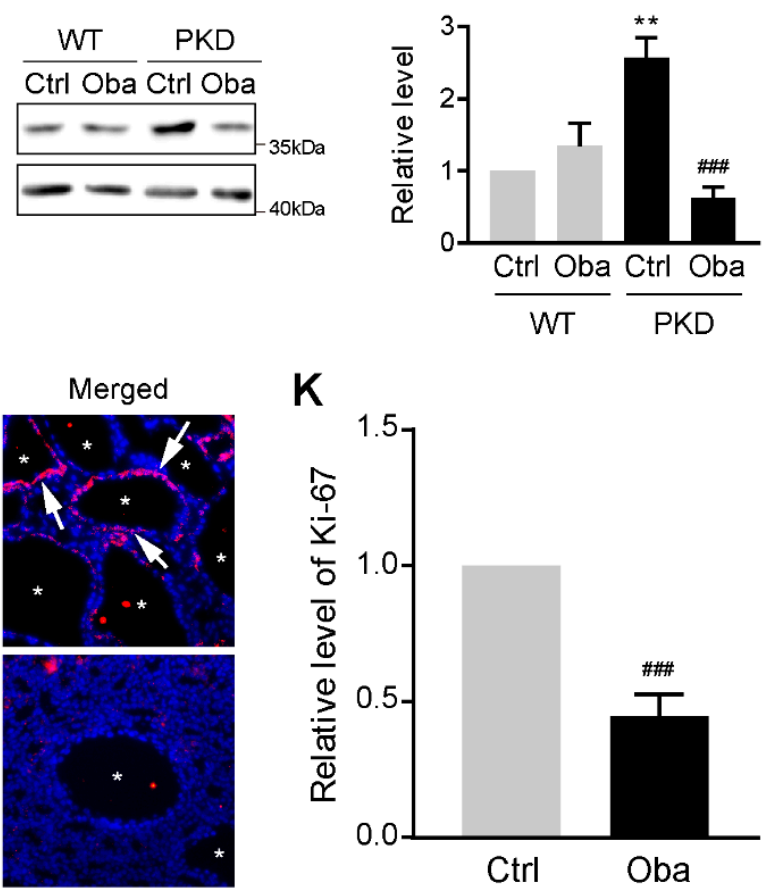

K

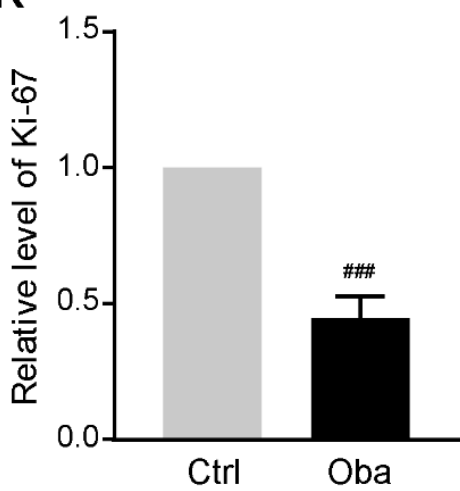

Figure 7. Inhibitory effect of obacunone on abnormal cell proliferation. (A) Representative Western blots of mTOR signaling pathway proteins in the kidneys of wild-type (WT) and PKD mice (PKD) treated without (Ctrl) or with obacunone (Oba). (B) Relative phosphorylation levels of mTOR protein. (C) Relative phosphorylation levels of S6 protein. (D) Representative Western blots of MAPK signaling pathway proteins. (E) Quantification of H-Ras protein expression levels. (F) Quantification of B-Raf protein expression levels. (G) Relative phosphorylation levels of ERK protein. (H) Representative Western blots of PCNA. (I) Quantification of PCNA protein expression levels. (J) Immunofluorescence staining of Ki-67 (red) and DAPI (blue) in the PKD kidneys. Arrows represent the Ki-67 positive cells, asterisks represent the cyst area. Bar $=50 \mu \mathrm{m}$. (K) Quantification of Ki-67 positive cells. Data are presented as means \pm SEM. $\mathrm{n}=6 .{ }^{*} p<0.05,{ }^{* *} p<0.01,{ }^{* * *} p<0.001$ versus WT-Ctrl. ${ }^{*} p<0.05$, \#\# $p<0.01, \# \#$, $p<0.001$ versus PKD-Ctrl. 


\section{Discussion}

The purpose of this study was to discover therapeutic drugs with strong efficacy and low side effects for ADPKD based on the importance of antioxidant signaling pathways in ADPKD progression. Obacunone was identified from a natural antioxidant compound library, which showed strong inhibitory effect on cyst development both in vivo and in vitro.

Obacunone is a triterpenoid limonoid compound, which is primarily found in the Rutaceae family plants, such as Phellodendron chinense and Tetradium ruticarpum [18,19]. Previous studies have reported that obacunone exhibits various biological properties, such as anti-inflammation [18], anti-tumor [33] and anti-virus [18,34]. Besides, the antioxidant capacity of it is most recognized. Obacunone acts as a NRF2 agonist by inhibiting its ubiquitination and promoting its nuclear translocation. It is reported that obacunone shows ROS scavenging ability in numerous oxidative stress-related diseases by activating NRF2 [17,19,35,36].

ADPKD is one of the oxidative stress-related diseases [13]. Oxidative stress elevates in renal tissues of ADPKD models as well as human patients [15,24,37-40]. Accumulation of reactive oxygen species is regarded as an important second hit factor that causes $P k d$ gene mutations and promotes cystogenesis [15,41]. Antioxidant defenses dysfunction is the main cause of oxidative stress in ADPKD. NRF2, as an important antioxidant protein, was found to be inactivated in ADPKD mouse models [24]. NRF2 knockout significantly accelerated renal cyst development [24]. In contrast, pharmacological activation of NRF2 effectively inhibited the progression of ADPKD [24]. Consistent with these investigations, we found that obacunone treatment for 3 days significantly promoted the nuclear translocation of NRF2 in PKD mice, which suppressed oxidative stress in ADPKD.

However, we did not find an activation effect of obacunone treatment for 5 days on NRF2 in PKD mice. In PKD mice, KEAP1 and GSK-3 $\beta$ are up-regulated, which promotes the degradation of NRF2 by activating KEAP1-CUL3 and $\beta$-TrCP-CUL1 pathways [24]. Obacunone treatment sharply promoted the nuclear translocation of NRF2 in the early stage of ADPKD, which resulted in the exhaustion of NRF2 at the same time. These processes prevented obacunone from activating NRF2 in PKD mice at postnatal day 5. Indeed, we did find down-regulation of total NRF2 expression in the obacunone-treated PKD mice at postnatal day 5 (data not shown).

NRF2 functions as a transcription factor that is responsible for the expression of antioxidants proteins and phase II detoxification enzymes [42], such as GPX4, HO-1 and NQO-1 [43]. These proteins show potent ROS scavenging abilities, and generally dysfunction in oxidative stress process [42]. In our study, we found that GPX4 was down-regulated as the decline of NRF2 activity in PKD kidneys, implying that GPX4 is involved in the progress of ADPKD. GPX4 is a key enzyme in regulating glutathione metabolism. Its dysfunction leads to glutathione metabolism disorder and directly promotes lipid peroxidation [43-45]. Lipid peroxidation elevation was found to promote cyst expansion in ADPKD [14,29]. In our study, it was found that obacunone reversed the down-regulation of GPX4, which restored normal glutathione metabolism and suppressed lipid peroxidation.

Lipid peroxidation mainly results from oxidative stress. Accumulated ROS attacks polyunsaturated fatty acid (PUFA)-containing phospholipids, producing large amounts of lipid peroxides (LPOs) that have adverse effects on cellular homeostasis [46]. MDA and 4-HNE are two most widely studied LPOs, which increase sharply in the kidney of ADPKD mouse model [29]. MDA and 4-HNE have been confirmed as the activators of mTOR and MAPK signaling pathways [28,30-32,46]. These two signaling pathways play critical roles in promoting ADPKD progression [1]. Activation of MAPK and mTOR was found in various experimental models as well as human patients $[1,47]$. Our results showed that obacunone reduced the MDA and 4-HNE contents in the PKD kidneys, followed by down-regulation of $\mathrm{mTOR}$ and MAPK signaling pathways. These processes significantly inhibited abnormal proliferation of renal cells.

Our study revealed an important pharmacological mechanism that obacunone regulates the NRF2/GPX4/LPOs pathway in ADPKD. The possible mechanism is diagramed 
in Figure 8, which suggests that obacunone promotes the nuclear translocation of NRF2, which facilitates the expression of GPX4. The up-regulation of GPX4 suppresses lipid peroxidation accompanied by a decline of 4-HNE and MDA, which down-regulates mTOR and MAPK signaling pathways. These processes finally retard abnormal cell proliferation and cyst expansion.

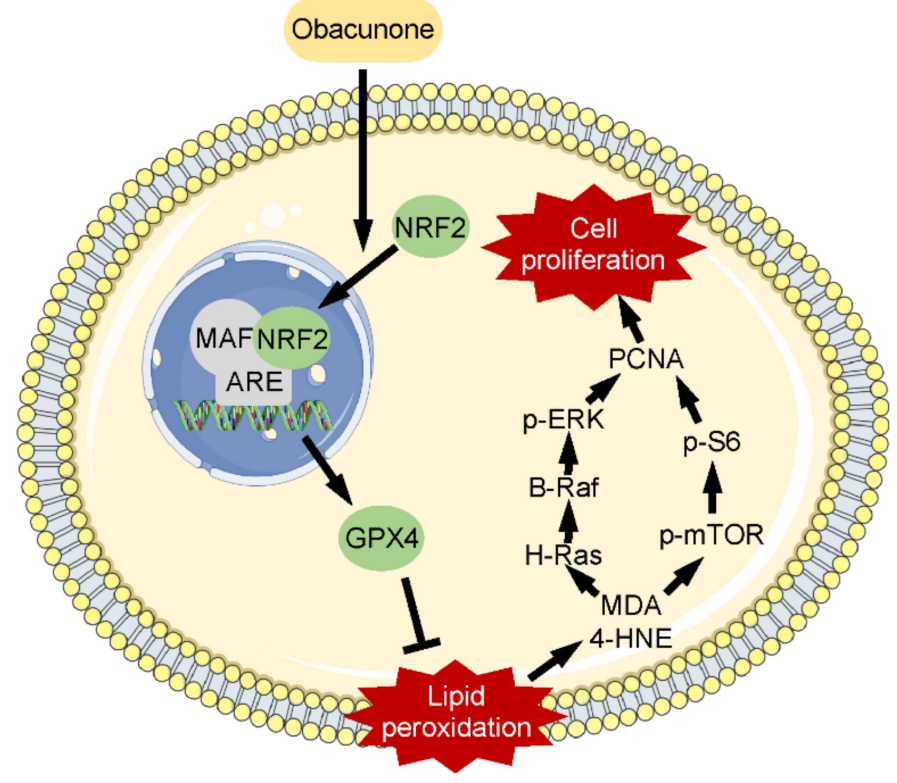

Figure 8. The suggested pharmacological mechanism of obacunone in ADPKD. See text for details.

However, some limitations of this study should be acknowledged. First, due to the limited experimental conditions, we did not generate a Pkd1 and Nrf2 double-knockout mouse model to verify the targeting effect of obacunone on NRF2. Second, we did not investigate the effect of obacunone on ferroptosis, even though lipid peroxidation is considered as the trigger of ferroptosis [44]. Elevated ferroptosis level was found in ADPKD mouse models [29], but how ferroptosis accelerates cyst expansion still needs to be clarified. Therefore, it will be a research interest for further studies.

\section{Conclusions}

Our study revealed the therapeutical effect of obacunone on ADPKD. It is the first study that suggests the application of obacunone on ADPKD treatment. The advantages of low cytotoxicity, easy availability and potent efficacy make obacunone ideal to be developed as a candidate therapeutic drug for ADPKD. The druggability of obacunone should be evaluated in further preclinical tests and clinical trials.

Author Contributions: Conceptualization, Z.Q., J.H. (Jinzhao He), H.Z. and B.Y.; Data curation, Z.Q.; Formal analysis, Z.Q.; Funding acquisition, H.Z. and B.Y.; Investigation, Z.Q., J.H. (Jinzhao He), G.S. and J.H. (Jiaqi Hu); Methodology, Z.Q., J.H. (Jinzhao He), X.L. and M.L.; Project administration, B.Y.; Resources, M.L. and B.Y.; Supervision, B.Y.; Validation, Z.Q. and G.S.; Visualization, Z.Q.; Writingoriginal draft, Z.Q.; Writing-review \& editing, G.S., J.H. (Jiaqi Hu) and B.Y. All authors have read and agreed to the published version of the manuscript.

Funding: This research was funded by the National Natural Science Foundation of China grants 81974083, 81620108029, 81330074, 81873597 and the Beijing Natural Science Foundation grant 7212151.

Institutional Review Board Statement: All procedures in this study were carried out in strict accordance with the recommendations in the Guide for the Care and Use of Laboratory Animals of China Association for Laboratory Animal Science. All animal care and protocols were approved by the Institutional Animal Care and Use Committee at the Peking University Health Science Center (Beijing, China, LA220354, 19 May 2020). All sacrifice was performed under anesthesia with pentobarbitone. 
Informed Consent Statement: Not applicable.

Data Availability Statement: All data produced in this study is provided in the manuscript.

Acknowledgments: We thank Peter Igarashi and Stefan Somlo for providing us with the Ksp-Cre and $P k d 1^{f l o x} / f l o x$ mice.

Conflicts of Interest: The authors declare no conflict of interest.

\section{References}

1. Cornec-Le Gall, E.; Alam, A.; Perrone, R.D. Autosomal dominant polycystic kidney disease. Lancet 2019, 393, 919-935. [CrossRef]

2. $\quad$ Bergmann, C.; Guay-Woodford, L.M.; Harris, P.C.; Horie, S.; Peters, D.J.M.; Torres, V.E. Polycystic kidney disease. Nat. Rev. Dis. Primers 2018, 4, 50. [CrossRef]

3. Arroyo, J.; Escobar-Zarate, D.; Wells, H.H.; Constans, M.M.; Thao, K.; Smith, J.M.; Sieben, C.J.; Martell, M.R.; Kline, T.L.; Irazabal, M.V.; et al. The genetic background significantly impacts the severity of kidney cystic disease in the Pkd1(RC/RC) mouse model of autosomal dominant polycystic kidney disease. Kidney Int. 2021, 99, 1392-1407. [CrossRef] [PubMed]

4. Cornec-Le Gall, E.; Torres, V.E.; Harris, P.C. Genetic Complexity of Autosomal Dominant Polycystic Kidney and Liver Diseases. J. Am. Soc. Nephrol. 2018, 29, 13-23. [CrossRef] [PubMed]

5. Padovano, V.; Podrini, C.; Boletta, A.; Caplan, M.J. Metabolism and mitochondria in polycystic kidney disease research and therapy. Nat. Rev. Nephrol. 2018, 14, 678-687. [CrossRef]

6. Liu, F.; Feng, C.; Shen, H.; Fu, H.; Mao, J. Tolvaptan in Pediatric Autosomal Dominant Polycystic Kidney Disease: From Here to Where? Kidney Dis. 2021, 7, 343-349. [CrossRef]

7. Chapman, A.B.; Devuyst, O.; Eckardt, K.U.; Gansevoort, R.T.; Harris, T.; Horie, S.; Kasiske, B.L.; Odland, D.; Pei, Y.; Perrone, R.D.; et al. Autosomal-dominant polycystic kidney disease (ADPKD): Executive summary from a Kidney Disease: Improving Global Outcomes (KDIGO) Controversies Conference. Kidney Int. 2015, 88, 17-27. [CrossRef] [PubMed]

8. Ong, A.C.; Devuyst, O.; Knebelmann, B.; Walz, G.; ERA-EDTA Working Group for Inherited Kidney Diseases. Autosomal dominant polycystic kidney disease: The changing face of clinical management. Lancet 2015, 385, 1993-2002. [CrossRef]

9. Gao, J.; Zhou, H.; Lei, T.; Zhou, L.; Li, W.; Li, X.; Yang, B. Curcumin inhibits renal cyst formation and enlargement in vitro by regulating intracellular signaling pathways. Eur. J. Pharmacol. 2011, 654, 92-99. [CrossRef] [PubMed]

10. Zhou, H.; Gao, J.; Zhou, L.; Li, X.; Li, W.; Li, X.; Xia, Y.; Yang, B. Ginkgolide B inhibits renal cyst development in in vitro and in vivo cyst models. Am. J. Physiol. Renal. Physiol. 2012, 302, F1234-F1242. [CrossRef]

11. Su, L.; Liu, L.; Jia, Y.; Lei, L.; Liu, J.; Zhu, S.; Zhou, H.; Chen, R.; Lu, H.A.J.; Yang, B. Ganoderma triterpenes retard renal cyst development by downregulating Ras/MAPK signaling and promoting cell differentiation. Kidney Int. 2017, 92, 1404-1418. [CrossRef] [PubMed]

12. He, J.; Zhou, H.; Meng, J.; Zhang, S.; Li, X.; Wang, S.; Shao, G.; Jin, W.; Geng, X.; Zhu, S.; et al. Cardamonin retards progression of autosomal dominant polycystic kidney disease via inhibiting renal cyst growth and interstitial fibrosis. Pharmacol. Res. 2020, 155, 104751. [CrossRef] [PubMed]

13. Andries, A.; Daenen, K.; Jouret, F.; Bammens, B.; Mekahli, D.; Van Schepdael, A. Oxidative stress in autosomal dominant polycystic kidney disease: Player and/or early predictor for disease progression? Pediatr. Nephrol. 2019, 34, 993-1008. [CrossRef]

14. Schreiber, R.; Buchholz, B.; Kraus, A.; Schley, G.; Scholz, J.; Ousingsawat, J.; Kunzelmann, K. Lipid Peroxidation Drives Renal Cyst Growth In Vitro through Activation of TMEM16A. J. Am. Soc. Nephrol. 2019, 30, 228-242. [CrossRef] [PubMed]

15. Ishimoto, Y.; Inagi, R.; Yoshihara, D.; Kugita, M.; Nagao, S.; Shimizu, A.; Takeda, N.; Wake, M.; Honda, K.; Zhou, J.; et al. Mitochondrial Abnormality Facilitates Cyst Formation in Autosomal Dominant Polycystic Kidney Disease. Mol. Cell. Biol. 2017, 37, e00337-17. [CrossRef] [PubMed]

16. He, J.; Zhang, S.; Qiu, Z.; Li, X.; Huang, H.; Jin, W.; Xu, Y.; Shao, G.; Wang, L.; Meng, J.; et al. Inhibiting Focal Adhesion Kinase Ameliorates Cyst Development in Polycystin-1-Deficient Polycystic Kidney Disease in Animal Model. J. Am. Soc. Nephrol. 2021, 32, 2159-2174. [CrossRef]

17. Xu, S.; Chen, W.; Xie, Q.; Xu, Y. Obacunone activates the Nrf2-dependent antioxidant responses. Protein. Cell 2016, 7, 684-688. [CrossRef]

18. Luo, X.; Yue, B.; Yu, Z.; Ren, Y.; Zhang, J.; Ren, J.; Wang, Z.; Dou, W. Obacunone Protects Against Ulcerative Colitis in Mice by Modulating Gut Microbiota, Attenuating TLR4/NF-kappaB Signaling Cascades, and Improving Disrupted Epithelial Barriers. Front. Microbiol. 2020, 11, 497. [CrossRef]

19. Huang, D.R.; Dai, C.M.; Li, S.Y.; Li, X.F. Obacunone protects retinal pigment epithelium cells from ultra-violet radiation-induced oxidative injury. Aging 2021, 13, 11010-11025. [CrossRef]

20. Cores, A.; Piquero, M.; Villacampa, M.; Leon, R.; Menendez, J.C. NRF2 Regulation Processes as a Source of Potential Drug Targets against Neurodegenerative Diseases. Biomolecules 2020, 10, 904. [CrossRef] [PubMed]

21. Rojo de la Vega, M.; Chapman, E.; Zhang, D.D. NRF2 and the Hallmarks of Cancer. Cancer Cell 2018, 34, 21-43. [CrossRef]

22. Jaramillo, M.C.; Zhang, D.D. The emerging role of the Nrf2-Keap1 signaling pathway in cancer. Genes Dev. 2013, 27, $2179-2191$. [CrossRef] 
23. Kansanen, E.; Kuosmanen, S.M.; Leinonen, H.; Levonen, A.L. The Keap1-Nrf2 pathway: Mechanisms of activation and dysregulation in cancer. Redox Biol. 2013, 1, 45-49. [CrossRef]

24. Lu, Y.; Sun, Y.; Liu, Z.; Lu, Y.; Zhu, X.; Lan, B.; Mi, Z.; Dang, L.; Li, N.; Zhan, W.; et al. Activation of NRF2 ameliorates oxidative stress and cystogenesis in autosomal dominant polycystic kidney disease. Sci. Transl. Med. 2020, 12, eaba3613. [CrossRef]

25. Dodson, M.; de la Vega, M.R.; Cholanians, A.B.; Schmidlin, C.J.; Chapman, E.; Zhang, D.D. Modulating NRF2 in Disease: Timing Is Everything. Annu. Rev. Pharmacol. Toxicol. 2019, 59, 555-575. [CrossRef] [PubMed]

26. Carlson, B.A.; Tobe, R.; Yefremova, E.; Tsuji, P.A.; Hoffmann, V.J.; Schweizer, U.; Gladyshev, V.N.; Hatfield, D.L.; Conrad, M Glutathione peroxidase 4 and vitamin E cooperatively prevent hepatocellular degeneration. Redox Biol. 2016, 9, 22-31. [CrossRef] [PubMed]

27. Wu, X.; Li, Y.; Zhang, S.; Zhou, X. Ferroptosis as a novel therapeutic target for cardiovascular disease. Theranostics 2021, 11, 3052-3059. [CrossRef]

28. Cui, W.; Wu, X.; Feng, D.; Luo, J.; Shi, Y.; Guo, W.; Liu, H.; Wang, Q.; Wang, L.; Ge, S.; et al. Acrolein Induces Systemic Coagulopathy via Autophagy-dependent Secretion of von Willebrand Factor in Mice after Traumatic Brain Injury. Neurosci. Bull. 2021, 37, 1160-1175. [CrossRef] [PubMed]

29. Zhang, X.; Li, L.X.; Ding, H.; Torres, V.E.; Yu, C.; Li, X. Ferroptosis Promotes Cyst Growth in Autosomal Dominant Polycystic Kidney Disease Mouse Models. J. Am. Soc. Nephrol. 2021, 32, 2759-2776. [CrossRef]

30. Lin, M.H.; Yen, J.H.; Weng, C.Y.; Wang, L.; Ha, C.L.; Wu, M.J. Lipid peroxidation end product 4-hydroxy-trans-2-nonenal triggers unfolded protein response and heme oxygenase-1 expression in PC12 cells: Roles of ROS and MAPK pathways. Toxicology 2014, 315, 24-37. [CrossRef] [PubMed]

31. Squadrito, F.; Minutoli, L.; Esposito, M.; Bitto, A.; Marini, H.; Seminara, P.; Crisafulli, A.; Passaniti, M.; Adamo, E.B.; Marini, R.; et al. Lipid peroxidation triggers both c-Jun N-terminal kinase (JNK) and extracellular-regulated kinase (ERK) activation and neointimal hyperplasia induced by cessation of blood flow in the mouse carotid artery. Atherosclerosis 2005, 178, 295-302. [CrossRef]

32. Zhang, Y.; Cui, Y.; Dai, S.; Deng, W.; Wang, H.; Qin, W.; Yang, H.; Liu, H.; Yue, J.; Wu, D.; et al. Isorhynchophylline enhances Nrf2 and inhibits MAPK pathway in cardiac hypertrophy. Naunyn-Schmiedeberg's Arch. Pharmacol. 2020, 393, 203-212. [CrossRef] [PubMed]

33. Murthy, K.N.; Jayaprakasha, G.K.; Patil, B.S. Cytotoxicity of obacunone and obacunone glucoside in human prostate cancer cells involves Akt-mediated programmed cell death. Toxicology 2015, 329, 88-97. [CrossRef] [PubMed]

34. Vardhan, S.; Sahoo, S.K. In silico ADMET and molecular docking study on searching potential inhibitors from limonoids and triterpenoids for COVID-19. Comput. Biol. Med. 2020, 124, 103936. [CrossRef] [PubMed]

35. Zhou, J.; Wang, T.; Wang, H.; Jiang, Y.; Peng, S. Obacunone attenuates high glucose-induced oxidative damage in NRK-52E cells by inhibiting the activity of GSK-3beta. Biochem. Biophys. Res. Commun. 2019, 513, 226-233. [CrossRef] [PubMed]

36. Bai, Y.; Wang, W.; Wang, L.; Ma, L.; Zhai, D.; Wang, F.; Shi, R.; Liu, C.; Xu, Q.; Chen, G.; et al. Obacunone Attenuates Liver Fibrosis with Enhancing Anti-Oxidant Effects of GPx-4 and Inhibition of EMT. Molecules 2021, 26, 318. [CrossRef]

37. Wang, D.; Strandgaard, S.; Borresen, M.L.; Luo, Z.; Connors, S.G.; Yan, Q.; Wilcox, C.S. Asymmetric dimethylarginine and lipid peroxidation products in early autosomal dominant polycystic kidney disease. Am. J. Kidney Dis. 2008, 51, 184-191. [CrossRef]

38. Zhang, L.; Li, L.X.; Zhou, J.X.; Harris, P.C.; Calvet, J.P.; Li, X. RNA helicase p68 inhibits the transcription and post-transcription of Pkd1 in ADPKD. Theranostics 2020, 10, 8281-8297. [CrossRef]

39. Fick-Brosnahan, G.M. Endothelial dysfunction and angiogenesis in autosomal dominant polycystic kidney disease. Curr. Hypertens. Rev. 2013, 9, 32-36. [CrossRef]

40. Chang, M.Y.; Ong, A.C.M. Targeting new cellular disease pathways in autosomal dominant polycystic kidney disease. Nephrol. Dial. Transplant. 2018, 33, 1310-1316. [CrossRef]

41. Qian, F.; Watnick, T.J.; Onuchic, L.F.; Germino, G.G. The molecular basis of focal cyst formation in human autosomal dominant polycystic kidney disease type I. Cell 1996, 87, 979-987. [CrossRef]

42. Osama, A.; Zhang, J.; Yao, J.; Yao, X.; Fang, J. Nrf2: A dark horse in Alzheimer's disease treatment. Ageing Res. Rev. 2020, 64, 101206. [CrossRef]

43. Du, Y.; Zhao, H.C.; Zhu, H.C.; Jin, Y.; Wang, L. Ferroptosis is involved in the anti-tumor effect of lycorine in renal cell carcinoma cells. Oncol. Lett. 2021, 22, 781. [CrossRef]

44. Yang, W.S.; SriRamaratnam, R.; Welsch, M.E.; Shimada, K.; Skouta, R.; Viswanathan, V.S.; Cheah, J.H.; Clemons, P.A.; Shamji, A.F.; Clish, C.B.; et al. Regulation of ferroptotic cancer cell death by GPX4. Cell 2014, 156, 317-331. [CrossRef] [PubMed]

45. Bersuker, K.; Hendricks, J.M.; Li, Z.; Magtanong, L.; Ford, B.; Tang, P.H.; Roberts, M.A.; Tong, B.; Maimone, T.J.; Zoncu, R.; et al The CoQ oxidoreductase FSP1 acts parallel to GPX4 to inhibit ferroptosis. Nature 2019, 575, 688-692. [CrossRef]

46. Ayala, A.; Munoz, M.F.; Arguelles, S. Lipid peroxidation: Production, metabolism, and signaling mechanisms of malondialdehyde and 4-hydroxy-2-nonenal. Oxid. Med. Cell. Longev. 2014, 2014, 360438. [CrossRef] [PubMed]

47. Nobakht, N.; Hanna, R.M.; Al-Baghdadi, M.; Ameen, K.M.; Arman, F.; Nobahkt, E.; Kamgar, M.; Rastogi, A. Advances in Autosomal Dominant Polycystic Kidney Disease: A Clinical Review. Kidney Med. 2020, 2, 196-208. [CrossRef] [PubMed] 